\title{
EL CONDE GARCÍA ORDÓÑEZ, RIVAL DEL CID CAMPEADOR. SU FAMILIA, SUS SERVICIOS A ALFONSO VI
}

\author{
JosÉ Ma CANAL SÁNCHEZ-PAGÍN \\ León
}

\section{SUMARIO}

I. Preliminares. 1. Fuentes de información: documentos, crónicas, romances. 2. Identificación del conde García Ordóñez.- II. Ascendencia del conde García.- III. Los dos matrimonios de García Ordóñez.- IV. García Ordóñez al servicio de Alfonso VI.- V. Descendencia del conde García Ordóñez: 1. Fernando García de Hita. 2. Mayor García de Carrión. 3. Elvira García. 4. Fortún Garcés Kaixal(?). 5. García Garcés de Aza.- VI Conclusiones.- Apéndice documental.

La obra de la reconquista del suelo patrio, invadido por los musulmanes, exigió al pueblo español muchos y grandes sacrificios. Desde la rebelión de don Pelayo en Covadonga hasta la conquista de Granada por los Reyes Católicos, no cesó esa importante tarea, al frente de la cual se hallaban los belicosos monarcas con todo su empuje y valor. Uno de estos fue Alfonso VI, "el conquistador de Toledo, el magnífico emperador". Continuando la obra de su activo padre, Fernando I, no solo conquistó la importante ciudad de Toledo, sino que ensanchó de modo notable el terreno conquistado y fortaleció las fronteras, que resistieron el mismo empuje de los almorávides, al final de su vida.

Para realizar esta importante obra, Alfonso tuvo que rodearse de hábiles caudillos, de sabios ministros, de aguerridos soldados. Algunos de esos caudillos tienen nombre bien conocido: Pedro Ansúrez, Martín Alfonso, Martín Flaínez, Alvaro Fáñez, García Ordóñez. 
En este breve artículo intentamos destacar la figura y la obra de este último, que dedicó toda su vida y su actividad a esa magna empresa de la reconquista, a las órdenes del rey, hasta derramar la última gota de su sangre en la batalla de Uclés (mayo 1108), al lado del hijo del mismo rey, el infante Sancho.

Hay que confesar en principio y paladinamente, que tanto la figura y la obra de Alfonso como las de su fiel vasallo el conde García, han sido ensombrecidas por la obra literaria que nos presenta de modo arbitrario y fantástico la figura y la obra de Rodrigo Díaz, el Cid Campeador. Don Ramón Menéndez Pidal, datando mal esa obra literaria, es decir, el Carmen Campidoctoris (hacia el año 1090), la Historia Roderici (hacia 1110), y el Cantar de Mio Cid (hacia 1140), y acentuando en demasía su carácter histórico, ha contribuido de modo notable a confirmar ese ensombrecimiento, esa deformación arbitraria de la figura del monarca y del conde García.

Esa delicada y difícil tarea de la datación de esas tres obras, y de descubrir la tendencia de sus autores a idealizar la figura del Cid, al mismo tiempo que a denigrar la figura del monarca y de su vasallo, va realizándose poco a poco por varios investigadores e historiadores. Comenzó a hacerse ya en vida del mismo ilustre catedrático, pero él apenas cedió en sus primeras posiciones, alegando además que en la Primera Crónica General de España, escrita bajo los auspicios de Alfonso X el Sabio, se consagraban muchas más páginas a Rodrigo Díaz que a Alfonso VI. En resumen, no admitió él que la mayor parte del Poema es de índole fantástica, y que por ende la citada Crónica contiene, en este caso, más invenciones retóricas que realidades históricas.

\section{PRELIMINARES}

\section{Fuentes de Información: Documentos, Crónicas, Romances}

Al disertar sobre los servicios de este conde al rey Alfonso VI, hemos de proceder con mucha cautela, ya que, como hemos indicado, la historia de esta época ha sido mezclada con la leyenda, y por tanto desfigurada. La primera crónica que nos interesa es la Historia Ruderici Campi Docti, que nos habla de la rivalidad y enemistad entre nuestro conde y Rodrigo Díaz, el Campeador. ¿Qué valor tiene esta crónica? Menéndez Pidal la creyó coetánea del héroe castellano y por tanto de un valor 
inestimable, como escrita por un testigo de los hechos. Este testigo habría sido un clérigo no castellano (aragonés, catalán, navarro?). Pero esta opinión no es hoy compartida por los estudiosos, y se opone a muchas afirmaciones del autor de esa Historia.

Si ese autor fue coetáneo, ¿por qué no supo identificar a la mujer del Cid? La supone él nieta o sobrina de Alfonso VI (neptis), pero esto se opone a la documentación. Ella era una noble dama asturiana sin relación de parentesco con el rey. ¿Por qué no conoce la genealogía del mismo Campeador, con certeza, y la da solo como probable, al decir: eius origo haec esse videtur? ¿Acaso no conocía el Cid su origen?.

No podemos exponer aquí este tema como se merece. Remitimos solo al estudio de Antonio Ubieto Arteta ${ }^{1}$. Este autor concluye que la Historia fue escrita entre 1137 y 1147, y creemos que su conclusión es acertada. Su autor pudo ser un monje de Ripoll, admirador del Campeador. En ese monasterio fue sepultada María, la hija del Cid, primera mujer de Ramón Berenguer el Grande, conde de Barcelona (1090-1131)2.

Pues bien, en esa Historia Ruderici se habla de dos encuentros entre nuestro conde y Rodrigo, y las dos veces para enaltecer el valor y la inteligencia de éste y empobrecer la figura de aquél. ¿Responden estos hechos a la realidad? Por lo que mira al primero, a la escaramuza de Cabra, si ello sucedió, debió tener muy poca importancia, dado que el rey de Granada, Abd Allah, no lo menciona en sus Memorias ${ }^{3}$.

'A. Ubieto ARTETA. El Cantar de Mio Cid y algunos problemas históricos. Valencia 1973, 228 p. En particular pp. 174-177.

Ya antes de la aparición de la obra de MENÉndez PIDAl, Cantar de Mio Cid, 3 vols. $1^{\text {a }}$ edición, Madrid 1980, el holándes D. DOZY (Recherches sur l'histoire de la litterature de l'Espagne pendant le Moyen Age, $3^{\mathrm{a}}$ ed. París-Leyde 1881, II, 73) situaba la composición de la Historia Ruderici hacia 1150. Con él coincidía A. Bonilla y SAN MARTín (Gestas del Cid Campeador, en "Bol. de la R. Academia de la Historia", 59, 1991, 170).

${ }^{2}$ De hecho, de ese monasterio procede el manuscrito más antiguo, s. XII-XIII, del Carmen Campidoctoris (ver Bonilla, Ibidem, p. 173). Y este poema pudo ser el complemento de la Historia Ruderici, como el Poema de Almaría es el de la Crónica del Emperador. Es más, hay razones para pensar que ambas obras, La Historia y La Crónica se deben al mismo autor. En ellas se observa el mismo peculiar estilo y los mismos modismos. Si ese monje de Ripoll llegó luego a ser obispo de Astorga, es otro problema.

${ }^{3} \mathrm{ABD}$ AllaH Rey de Granada. El Siglo XI en primera persona. Las Memorias de Abd Allah, último rey zirí de Granada, destronado por los Almorávides (1090), traducidas por E. LévyProvenzal y E. García Gómez, Madrid 1980, 344 pp. 
Y por lo que mira al segundo hecho, al encuentro de la Rioja, es evidente que el autor carga las tintas para denigrar al conde y exaltar al héroe.

Respecto a la acentuada envidia de Alfonso VI y de sus cortesanos ante los triunfos del Cid, habríamos más bien de hablar de rabia ante la indisciplina constante del revolucionario Campeador. De hecho los que ayudaron al rey, con método y con disciplina, en las campañas de reconquista y sobre todo en la conquista de Toledo y de las villas circundantes, no fue Rodrigo, sino los condes y magnates de la corte: Pedro Ansúrez, Martín Alfonso, Martín Flaínez, Alvaro Fáñez, García Ordóñez, etc.

Otra fuente de información sobre el conde es el Cantar de Mio Cid, que Menéndez Pidal creyó compuesto hacia 1140-1150, y por tanto de mucho valor histórico. Pero, ¿es esto así? De ningún modo. Los críticos de hoy, y con mucho acierto, sitúan la composición de este poema a fines del siglo XII o comienzos del XIII. En vano se esforzó el citado polígrafo en demostrar la historicidad de este Cantar: hay en él muchos datos del todo fabulosos, y personajes del todo ficticios. Hoy ya nadie cree en la jura de Santa Gadea, ni en la realidad de las bodas de los Infantes de Carrión ${ }^{4}$.

Por lo que mira a la documentación, hay que precisar que Menéndez Pidal alega en su Cartulario Cidiano algunos documentos falsos. Uno de ellos es la carta de arras que el Cid da a su esposa Jimena el año 1074. Es un documento inventado en la segunda mitad del siglo XII, cuando la euforia cidiana llegaba a su colmo ${ }^{5}$.

\section{Identificación del conde García Ordóñez}

No ha sido tarea fácil identificar a nuestro conde, una vez que la Primera Crónica General nos lo presentó bajo tantos nombres y autor de tantas peripecias. Sus nombres: el conde García de Cabra, el conde García de Nájera, el Crespo de Grañón, etc. Sus peripecias: él acompaña a Sancho

\footnotetext{
${ }^{4}$ Ver las modernas ediciones del Poema de Mio Cid, por Colin SMITh (Madrid 1981) y por $M^{\text {a }}$ EUGENIA LACARRA (Madrid 1983)

Escribe ésta: "En cuanto a la datación del Poema, muchos estudios recientes coinciden en proponer una fecha cercana a 1207, fecha constatada en el manuscrito" (Ibidem, p. 27).

${ }^{5}$ Algunos modernos autores siguen proponiendo como auténtico ese documento: D. Mansilla Reoyo, Catálogo documental del A. Catedral de Burgos, Madrid 1971, p. 26. MARTínEZ DíEZ, Orígenes familiares de Santo Domingo, en C. Aniz Iriarte, OP, (ed.), Santo Domingo de Caleruega. Jornadas de E. Medievales, Salamanca 1994, 173-228.
} 
II en el cerco de Zamora, él cae en la ira del rey Alfonso VI y se pasa a los moros, él muere en la batalla de Uclés amparando al infante Sancho Alfonso, etc. ${ }^{6}$.

Aún el mismo intuitivo fray Prudencio de Sandoval no supo deshacerse de esta confusión que reina en las Crónicas generales, llegando a declarar: "Y no sé si acertaríamos más diciendo que este don García de Cabra fuese diferente de don García Ordóñez, y ambos enemigos del Cid"?.

Esta confusión se acentúa en los tres grandes genealogistas, que son Pellicer, Suárez de Alarcón y Salazar y Castro. Los tres distinguen al de Cabra del de Nájera. Suárez de Alarcón dice: "El conde don García Ordóñez, el primero del nombre, fue hijo del conde Ordoño Ordóñez, y nieto del infante Ordoño y de la infanta Cristina. Estuvo casado con la infanta Elvira Fernández, y murió en Rueda el año 1083. El segundo conde García Ordóñez fue el de Nájera, casado con la infanta Urraca de Navarra, muerto en Uclés". Fue mérito de Menéndez Pidal haber eliminado esta confusión identificando al de Cabra con el de Nájera ${ }^{8}$.

\section{ASCENDENCIA DEL CONDE GARCÍA}

El apellido del conde indica ya el nombre del padre: Ordoño. Y la documentación auténtica nos añade que ese personaje se llamaba Ordoño Ordóñez. La primera mención la vemos en un pergamino de la catedral de León. Con fecha 7 de enero de 1043, el rey Fernando I y su esposa doña Sancha hacen una donación a la iglesia de Santa María, y en ella se hace constar que el alférez regio era Ordoño Ordóñez: Ordonio Ordoniz armiger ${ }^{9}$.

\footnotetext{
${ }^{6}$ Primera Crónica General de España, editada por R. MEnÉndez PIDAl, $2^{\mathrm{a}}$ ed. Madrid 1977. Ver Indice general.

${ }^{7}$ Fray P. DE SANDoval, OSB, Historia de los reyes de Castilla y de León, Madrid 1792, ed. Benito Cano, 178.

${ }^{8} \mathrm{~A}$. SUÁREZ DE ALARCÓN, Relaciones genealógicas de la Casa de los Marqueses de Trocifal, Madrid 1656, 109 y ss. J. PELLICER, Informe de la Casa de Sarmiento de Villamayor, Madrid 1663, 37-38. L. DE SAlazAr y CASTRO, Historia gen. de la Casa de Lara, Madrid 1696, Libro 19, cap. 2, tomo III, p. 309-314.

R. MEnÉndez PIDAl (Cantar de Mio Cid, II, 705) escribe: "Esta doble denominación no nos permite pensar, como generalmente se cree, que don García de Cabra sea distinto del conde de Nájera".

${ }^{9}$ J.M. Ruiz AsEnCio, Colección documental del archivo de la catedral de León, León 1990, IV, p. $172, \mathrm{n}^{\circ} 1007$.
} 
A continuación, este personaje aparece casi solo en la documentación castellana, por eso vamos a limitarnos a recoger las menciones que de él se hacen en el auténtico Cartulario de Cardeña, escrito a finales del siglo XI:

El 1 de julio 1047 el magnate Salvador González hace una donación al monasterio. Al final de la lista de los testigos, entre los que se halla Rodrigo Díaz (casi seguro el Cid), figura el alférez real: Ordonio Ordoniz armiger regis $c f$.

El 29 de octubre 1047 hace otra donación el magnate Nuño Alvarez. Está confirmada por magnates de alta categoría, y entre ellos está Ordonio Ordoniz testis.

El 3 agosto 1048 hacen otra donación las dos hermanas Eilo y Elvira. Son pocos los confirmantes, pero entre ellos está Ordonio Ordoniz $r b$.

El 31 agosto 1050 hacen una donación los reyes Fernando I y su esposa Sancha. Confirman los condes de la corte, algunos magnates castellanos y al final está Ordonio Ordoniz testis.

El 1 julio 1060 hace la donación Nuño Núñez. Pocos pero relevantes testigos y entre ellos está Ordonio Ordoniz testis.

El 25 julio 1063 el mismo personaje hace otra donación, confirmada nada menos que por el infante don Sancho, por el magnate Diego Alvarez, Ordoño Ordóñez, y su hijo García: Garsea filius eius rb., y otros tres testigos.

El 16 julio 1064 donan los reyes Fernando I y Sancha, y entre una docena de magnates castellanos está Ordoño Ordóñez.

El 1 diciembre 1065 dona Armentero Velasco, y entre los testigos se halla Ordoño Ordóñez.

El 25 de febrero 1066 se halla también el mismo en una donación privada.

El 8 febrero 1068 hace donación el rey Sancho II, confirman diez magnates castellanos, y entre ellos en séptimo lugar está Ordonio Ordoniz $r b$. No está el Campeador.

El 26 marzo 1071 el mismo rey dona. Confirman quince magnates, y en quinto lugar está Ordonio Ordoniz testis. Se halla el padre de Alvaro Fáñez, Fanni Fanniz, pero no el Campeador.

El 27 mayo 1072 hacen la donación varios magnates, y entre ellos Ordoño Ordóñez, con su mujer Anderkina, García Ordóñez (su hijo), etc.

El 8 diciembre 1072 hace la donación Alfonso VI, el nuevo rey de Castilla, confirman los condes de la corte y otros magnates castellanos, en 
total catorce. El sexto lugar lo ocupa Ordoño Ordóñez, el décimo su hijo García Ordóñez, el duodécimo Rodrigo Díaz (el Cid).

De todos estos documentos y diplomas podemos deducir: 1) que Ordoño Ordóñez ocupó el nobilísimo cargo de alférez real los años 1043 y 1047, y probablemente también en los años intermedios; 2) Que su mujer se llamaba Anderkina; 3) Que hijo suyo es García Ordóñez, el futuro conde. Por otro documento de Cardeña sabemos también que tuvo otros cuatro hijos, dos varones, Rodrigo y Diego, y des hembras, Teresa y Argilo ${ }^{10}$.

Ahora nos preguntamos sobre la ascendencia de Ordoño Ordóñez. ¿Era castellano o leonés? ¿Era un simple magnate o corría por sus venas sangre real? Es una cuestión delicada y no existe acuerdo sobre ello entre los modernos historiadores. Hasta los comienzos del siglo XX era opinión común y corriente que Ordoño descendía de la familia real leonesa. Fray Prudencio de Sandoval es el que más insiste sobre ello. Hace a Ordoño hijo del infante Ordoño y nieto de Alfonso IV el Monje. Los genealogistas Pellicer, Suárez de Alarcón y Salazar y Castro lo suponen hijo de la infanta Cristina y de su marido Ordoño Ramírez el Ciego y nieto de Vermudo II ${ }^{11}$.

Menéndez Pidal rechaza esta opinión, como carente de fundamento, y afirma sencillamente que Ordoño era uno más de los magnates castellanos de aquel tiempo ${ }^{12}$. ¿Razones de unos y de otros? Prueba concreta y documental no existe por ningún lado, pero podemos imaginar que Sandoval y los que le siguen intuyeron una razón oculta, y es que el matrimonio de García Ordóñez con una infanta navarra no se explica sino en el caso de que su padre, Ordoño, fuera de la casa real leonesa. Por su parte Menéndez Pidal no atendió a esta razón y pensó que Ordoño tenía que ser castellano por aparecer siempre entre la nobleza castellana. Y este fue su fallo. No

${ }^{10}$ L. Serrano (Ed.), Becerro gótico de Cardeña, Silos-Valladolid 1910, pp. 86, 182, 87, 43, 98, 62, 196, 277, 281, 160, 243, 100, 268.

"SANDOVAl, $O b$. cit. en nota 7, p. 76. Para los demás genealogistas, las obras citadas en la misma nota 7 .

${ }^{12}$ MenÉndez Pidal, La España del Cid, $7^{\text {a }}$ ed. Madrid 1969, II, 715. Lo mismo opina G MARTínez DíEZ en el art. citado supra en nota 5, p. 179. Por su parte J. DE SALAZAR ACHA (El linaje castellano de los Castro en el siglo XII, en "Anales de la R. Academia matritense de Heráldica y Genealogía”, I, 1991, 56-57) afirma menos categóricamente que Ordoño Ordóñez podría ser hijo de Ordoño Fáfilaz, y biznieto de Diego Muñoz, conde de Carrión y Saldaña. 
atender a la categoría social de García Ordóñez, categoría que en aquel tiempo se medía en primer lugar por la nobleza de la sangre ${ }^{13}$.

Opinamos pues que Ordoño Ordóñez era hijo de la infanta Cristina Vermúdez y de su marido Ordoño Ramírez. Don Pelayo, obispo de Oviedo, afirma en su crónica que esta infanta tuvo tres hijos: Alfonso, Sancha y Aldonza Ordóñez. El Tudense le corrige de este modo. Sus hijos fueron Alfonso, Ordoño, Pelaya y Aldonza Ordóñez. El hecho de no conocer don Pelayo a nuestro Ordoño Ordóñez pudo ser que este abandonase muy joven la región asturiana para ponerse al servicio de Fernando I, e instalarse definitivamente en Castilla ${ }^{14}$.

El mismo monarca pudo facilitarle el honroso matrimonio con una rica y noble dama castellana, Anderkina, hacendada en torno a las villas de Castrogeriz y de Palenzuela del Conde, como se desprende de las heredades de sus hijos y de sus nietos.

\section{LOS DOS MATRIMONIOS DE GARCÍA ORDÓÑEZ}

García Ordóñez nació en Castilla, se crió en Castilla, pero su primera mujer no fue castellana, sino navarra y se llamaba Urraca García. Era hija del rey García Sánchez de Navarra (1035-1054) y de su esposa la catalana Estafanía. Este matrimonio debió verificarse entre los años 1072 y 1076, ya que un documento de Cardeña de ese año 1072 García Ordóñez aparece aún célibe y el año 1076 goza ya de la dignidad condal, dignidad que solía concederse a los ya unidos en matrimonio ${ }^{15}$.

De este primer matrimonio conocemos tres hijos: Fernando García, Mayor García y Elvira García, de los que más tarde hablaremos. No

\footnotetext{
${ }^{13}$ A. SÁNCHEZ CANDEIRA, en un bien razonado estudio (La reina Velasquita de León y su descendencia, en "Hispania", 10, 1950, 449-505, en particular p. 488) vuelve a sostener la antigua sentencia de los grandes genealogistas, según los cuales Ordoño Ordóñez era nieto de la infanta Cristina Vermúdez. Basa su argumentación en cuatro razones que puede ver el lector en ese estudio, nota 104, pp. 488-489.

${ }^{14}$ Pelayo Obispo de Oviedo, Crónica, ed. Flórez, E.S., 14, 468. Lucas de Tuy, Crónica del mundo, ed. Schott, Hispania Illustrata, IV, 89.

${ }^{15}$ Becerro gótico de Cardeña, p. 268. En la Carta de arras que el Cid otorga a Jimena, fecha 19 julio 1074 (ed. La España del Cid, II, 837) aparecen como fiadores los condes Pedro Ansúrez y García Ordóñez, pero ya hemos dicho que esta carta no nos merece fe alguna. Ni García Ordóñez era conde en aquel año, ni él podía codearse con el veterano Pedro Ansúrez. Era solo un cortesano de segunda categoría.
} 
conocemos la fecha de la muerte de esta primera esposa, Urraca. Nos consta que vivía el año 1089, cuando los vecinos de Matute y de Tobía elogian a los condes de Nájera, sus señores: "Inclito et honorato a Deo et hominibus, et sub gratia Dei et Alfonsi regis, domno comite Garsea et nobilissima domna Urraka comitissa, dominantibus Naggera" ${ }^{16}$.

El segundo matrimonio del conde García se verificó hacia el año 1105 o algo antes. Sabemos que en el curso del año 1106 el conde bautizó un hijo en la iglesia de San Millán de la Cogolla, y ese niño debió ser ya hijo de este segundo matrimonio. Con mucha probabilidad fue García Garcés, el futuro señor de Aza. Por el Toledano sabemos que esta segunda mujer se llamaba Ava, nombre que luego ella cambió por el de Eva. ¿A qué linaje pertenecía esa señora? La opinión corriente, iniciada por el conde Pedro Barcelos en la primera mitad del siglo XIV y continuada hasta hace pocos años, afirmaba que ella era hija del conde gallego Pedro Froilaz de Trava, pero es muy poco creíble, dado que la familia de este conde nos es de sobra conocida por los documentos de Sobrado ${ }^{17}$.

Por eso, con mucha razón Jaime de Salazar Acha, en un estudio muy documentado, sugiere que esta señora Ava procedía de la región pirinaica, donde es frecuente tal nombre, y con el profesor Szaboles de Vajay piensa que "tal vez esta misteriosa Eva fuera hija de Aimerico II, vizconde de Rochechouart, cuya madre se llamaba Eva"18.

Admitamos que esta noble dama proviniese de algún condado pirinaico, como de ellos provino también otra Ava, la mujer del conde castellano García Fernández, llamada "la Condesa traidora", pero precisar de qué condado provino es aventurado, ya que, como hemos dicho, en varios de ellos aparece tal nombre.

La condesa se llamaba Ava/Eva, y el primer hijo de su segundo matrimonio se llamó Amalrico, Malrico (no Aimerico), y tales nombres

\footnotetext{
$\mathrm{n}^{\circ} 274$.

${ }^{16}$ L. Serrano, OSB (Ed.), Cartulario de San Millán de la Cogolla, Madrid 1930, p. 277,

${ }^{17}$ Serrano, Cartulario de San Millán, p. 295, no 292. El Conde Pedro Barcelos, al mencionar a los Lara, afirma que el conde Pedro González de Lara estuvo casado con "la condesa doña Eva, hija del conde don Pedro Fernández de Trava" (Libro de los Linajes, ed. PMH, SS. I, Lisboa 1856, 261), y luego afirma que la misma Eva se casó con García Garcés de Aza, hijo del conde García de Nájera, cuando, según la historia, este García Garcés de Aza fue hijo de ese conde y de su mujer la condesa Eva o Ava.

${ }^{18}$ SAlAZAR ACHA, art. citado supra en nota 12, p. 54, nota 106
} 
aparecen también en los condados catalanes. Pongamos un ejemplo, con los condes de Ampurias:

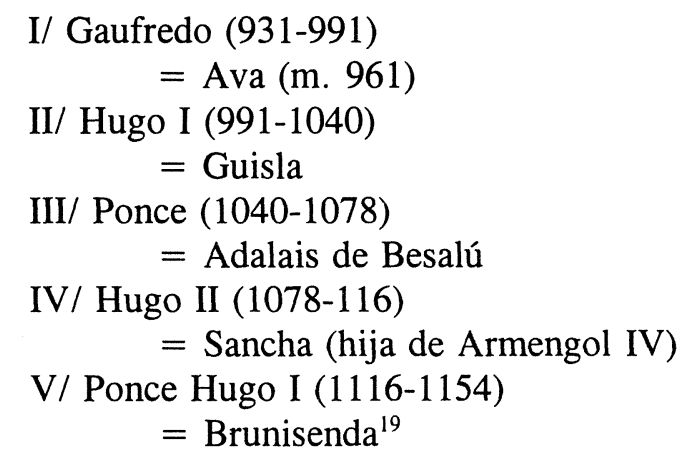

Nada extrañaría que la mujer del conde García fuera hija de ese Hugo II y de su mujer Sancha de Urgel, y que le hubiesen dado el nombre de una abuela. Es una mera conjetura. En favor de esa teoría, que la condesa Ava/Eva procediese de algún condado pirenáico está el hecho que su hijo Manrique o Amalrico se casase con la vizcondesa de Narbona, Ermensenda, pariente quizás de su propia madre ${ }^{20}$.

\section{GARCÍA ORDÓÑEZ AL SERVICIO DE ALFONSO VI}

Ya hemos indicado que la primera memoria documental de García aparece el año 1063 en una donación privada de Cardeña. En ese año debía él ser aún muy joven, quizás recién armado caballero, unos veinte años de edad. Comenzaba su carrera de soldado, de vasallo de los reyes Fernando I, Sancho II, Alfonso VI, ocupando aún en la corte un puesto muy humilde

\footnotetext{
${ }^{19}$ Sobre los condes de Ampurias: Salazar y Castro, Lara, III, 38, libro 16, cap. 7. S. SOBREQUES, Els Barons de Catalunya, Barcelona 1980, 7-14.

En JAIME VILlanUeVa (Viage literario, XIII, Gerona, p. 252) puede verse un documento del año 980 en que confirman: Aimericus levita, y Amalricus subdiaconus. Son dos nombres distintos. En el mismo autor (Viage, XIII, pp. 58, 65, 247) aparecen el conde de BesalúCerdanya, Óliba con su madre la condesa Ava, año 968.

${ }^{20} \mathrm{La}$ condesa Eva (Ava) aparece en una donación suya a la iglesia de Burgos, año 1147: "Ego comitissa Eva, una cum filiis meis" (SERRANo, Obispado de Burgos, III, p. 186, n 107).

Para los vizcondes de Narbona: Salazar y Castro, Casa de Lara, I, 109 y ss. Y una tabla en p. 131.
} 
al lado de su padre Ordoño. No comenzará a brillar sino unos diez años más tarde, cuando Alfonso VI le nombra su alférez, en 1074, es decir, cuando su padre ya había muerto. En una donación privada al monasterio de Sahagún, 20 febrero 1074, se enumeran los personajes de la curia regia, y después de los condes y magnates más fieles del "nuevo rey" está el alférez: Garcia Ordoniz armiger regis $c f .{ }^{21}$.

Atendiendo a los diplomas auténticos, solo dos años más tarde viene condecorado con la dignidad condal, y con la tenencia y el señorío de Nájera. El cargo de alférez pasa a Fernando Flaínez, a Rodrigo González, y por fín en 1081 a su hermano Rodrigo Ordóñez ${ }^{22}$.

En el concilio de Burgos (o de Sahagún) del 8 de mayo de 1080, en que se determina el cambio de rito litúrgico, García Ordóñez forma parte de la corte, pero, como es natural, en un puesto secundario, después de los grandes condes Pedro Ansúrez, Martín Alfonso, Rodrigo Díaz y su hermano Fernando Díaz de Asturias de Oviedo, Rodrigo Muñoz de Asturias de Santillana, y los gallegos Rodrigo Ovéquiz, Nuño Mitiz y su hermano Fernando Mitiz $^{23}$.

A partir de esta fecha García formará parte de la curia regia, e irá ascendiendo gradualmente a medida que los primeros condes van envejeciendo o muriendo. Solo en 1100 ocupará uno de los primeros puestos, con los condes Martín Flaínez (leonés) y Gómez González (castellano).

En las relaciones de la batalla de Sagrajas (Sacralias, 1086) no aparece su nombre, pero está en cambio el de su hermano Rodrigo, que ocupa el cargo de alférez. De todos modos no pudo faltar nuestro conde en tan importante encuentro, al lado de Alvaro Fáñez y de otros capitanes del ejército cristiano.

Algunos cronistas y el mismo Tudense, haciéndose eco de los cantares de gesta, ponen por estas fechas el paso de García a las filas del ejército moro, al servicio de los reyes taifas, una vez que había caído en la desgracia o ira regia de Alfonso VI, pero esto es una pura invención de los juglares. Por la documentación nos consta de modo bien claro que el conde García estuvo siempre al lado del rey cristiano, como fiel vasallo.

\footnotetext{
${ }^{21}$ M. HERRERO DE LA FUENTE, Colección diplomática del monasterio de Sahagún, León, 1988 , III, p. $10, \mathrm{n}^{\circ} 733$.

${ }^{22}$ Ver infra nota 25.

${ }^{23}$ HerRero de la Fuente, Ibidem, p. $70, \mathrm{n}^{\circ} 781$.
} 
En confirmación de esto, vamos a poner aquí la lista de memorias de nuestro conde que nos ofrece el Cartulario de San Millán de la Cogolla:

El 16 de junio de 1074 el nuevo rey Alfonso VI confirma las donaciones del monasterio. Confirman unos pocos condes y magnates, y al final aparecen Rodrigo Díaz (el Cid) y García Ordóñez.

El año 1077 Alfonso VI hace una donación: el primer confirmante es "el conde García Ordóñez en Nájera", y le sigue el conde Gómez (González) en Bureba.

El 11 noviembre 1084 en una donación privada, confirman los señores de Oca, de Grañón, y el de Nájera que es nuestro conde.

El año 1086 en una donación de un presbítero, confirma G.O.

El 21 julio 1087, en un diploma de Alfonso VI confirma G.O.

El mismo año 1087 en otra donación de Alfonso VI está G.O.

En el año 1088 en una donación privada está G.O.

En un documento de 1089 se hace elogio de los condes de Nájera, García Ordóñez y (la infanta) Urraca, "señores de Nájera".

El año 1091 Alfonso VI hace donación, y confirma el conde.

En una donación privada del año 1092 está G.O.

El 7 abril 1098 dona Alfonso VI, y confirma el conde.

En una donación de 1102 confirma el conde.

En una donación de Alfonso VI año 1106, se menciona el bautizo de un hijo del conde, y se afirma que él "domina en Calahorra y en Nájera" ${ }^{24}$.

Notemos que García Ordóñez tuvo el señorío y la tenencia de la región de Nájera desde 1076 hasta su muerte, 1108. Su esposa la infanta Urraca tenía ya el señorío de tres villas, que su madre la reina Estefamía, 1060, le había dejado en testamento, que eran Alberite, Lardero y Mucrones ${ }^{25}$.

\footnotetext{
${ }^{24}$ Serrano, Cartulario de San Millán, años citados. Sobre el paso de García al servicio de los taifas: Lucas de Tuy, Crónica, ed. cit. p. 101, 48. Poco antes había dicho (líneas 24-26) que Alfonso VI, para congraciarse con su vasallo "el conde castellano, García de Cabrera" (!), le concedió por esposa a su hermana Elvira Fernández. Rodrigo Jiménez de Rada (El Toledano), De Rebus Hispaniae, libro 6, cap. 31, ed. M.D. Cabanes, Valencia 1968, p. 144. Por otro lado afirma que "el conde García era el nutricio del infante Sancho Alfonso" (Ibidem, libro 7, cap. 15 , p. 159).

La Primera Crónica General, ed. citada, 558 b, 20, sigue los fabulosos relatos de los dos cronistas arriba citados, amalgamandolos como le es posible. Por ende se explica la confusión que reina entre los historiadores y genealogistas de los siglos XVI y XVII.

${ }^{25}$ Rodríguez de Lama, Colección de la Rioja, II, $2^{\mathrm{a}}$ ed., p. $69, \mathrm{n}^{\circ} 18$.
} 
La Rioja había pertenecido desde antiguo al reino de Navarra, pero al morir Sancho III el de Pañalén, 1076, Alfonso VI la ocupó y la anexionó a sus dominios.

La tenencia de esta zona ocasionó al conde García varios sinsabores. Lindaba por el oriente con el reino moro de Zaragoza, y era natural que los musulmanes molestasen por este lado. La Historia Ruderici menciona las incursiones que, de parte del taifa moro, hizo aquí el Campeador: "Propter comitis (Garsias) inimicitiam, et propter eius dedecus, praefatam terram Rodericus flamma ignis incendit, destruxit et devastavit"26.

En el Carmen Campidoctoris se alude también a este hecho:

\section{Ad quam, Garsiam, comitem superbum, rex praenotatus misit debellandum ${ }^{27}$.}

No estamos seguros, pero es posible que también Alfonso I el Batallador, rey de Aragón y de Navarra (1104-1134), alimentase pretensiones sobre esta tierra. El caso es que en mayo de 1107, Alfonso VI se dirigía con un poderoso ejército super Vascones et Aragonenses. ¿Sería con el fin de defender esta zona de la Rioja?.

Los dos diplomas que dan noticia de esa expedición señalan los contingentes que componían ese ejército, lo cual es interesante, porque debió ser el mismo que en la primavera siguiente se enfrentó en Uclés con los Almorávides. Son las fuerzas reunidas de todo el reino castellano-leonés, presididas por sus respectivos capitanes.

Después de la familia real y de los obispos, viene nuestro conde, seguido de los condes Gómez González de Bureba, Rodrigo Muñoz de Cantabria, Martín Flaínez de León, y luego Gómez Martínez (el hijo del conde Martín Alfonso), el príncipe de la milicia toledana que era Fernando Téllez, Fernando García (hijo de nuestro conde), alcalde de Medinaceli y de Guadalajara, Alvaro Fáñez, señor de Zorita y de Santa Veria, y el ayo y pedagogo del infante Sancho que era Pelayo Fernández ${ }^{28}$.

\footnotetext{
${ }^{26}$ Historia Roderici, no 50, ed. MenÉndez PIDAL, La España del Cid, II, 956, 22.

${ }^{27}$ Carmen Campidoctoris, 77, ed. MENÉNDEZ PIDAL, Ibidem, II, 884.

${ }^{28}$ El diploma del 8 de mayo en: SÁNCHEZ ALBORNOZ, Despoblación y repoblación del valle del Duero, Buenos Aires 1966, 387-389. J.A. García Luján, Privilegios reales de la cat. de Toledo, Toledo 1982, II, p. 22, $\mathrm{n}^{\circ}$ 3. Regesto en: F.J. HERnÁNDEZ, Los Cartularios de Toledo. Catálogo documental, Toledo 1985, 854 pp.
} 
¿Participó de veras García Ordóñez en la batalla de Uclés? Habría motivo para dudar de ello, si prestamos fe a las crónicas. Tanto el Tudense, como el Toledano, como la Primera Crónica General, afirman que en esa batalla murieron los condes García Fernández y Martín y otros condes, junto con el infante Sancho Alfonso, pero no mencionan a García Ordóñez. La Crónica Najerense en cambio dice que en esa batalla murió el infante Sancho y con él "el conde García de Grañón, llamado el Crespo"29.

Como no existía ningún conde García Fernández en este reino por este tiempo y el conde García Ordóñez desaparece de la documentación a partir de esta fecha, hay que concluir que fue nuestro conde quien murió con el infante. Por los mismos motivos deducimos que murieron también en ella el conde Martín Flaínez de León, y Gómez Martínez, el hijo del conde Martín Alfonso. Por el documento citado arriba, sabemos también que el ayo del infante no era el conde García sino Pelayo Fernández ${ }^{30}$.

El conde castellano Gómez González de Bureba y el capitán Alvaro Fáñez, que sin duda participaron también en este combate, lograron escapar y salvar su vida.

\section{DESCENDENCIA DEL CONDE GARCÍA ORdóÑEZ}

Muy mal informados estamos sobre esta descendencia. De los hijos del primer matrimonio con la infanta navarra, nada nos dicen las crónicas. De los del segundo matrimonio, es el Toledano quien nos ofrece un dato verídico e interesante: hijo de García y de Ava fue García Garcés el de Aza, que fue luego ayo de Alfonso VIII" 31 .

El diploma del 14 mayo: A. López Ferrero, Historia de la I. de Santiago, III, Apéndice, p. 70, $\mathrm{n}^{\circ}$ 23. El comentario en: SÁNCHEZ ALBORNOZ, ¿Dónde vas, Alfonso VI? en "Miscelánea de estudios históricos”, León, 1970, 457-464.

${ }^{29}$ Crónica Najerense, 56, ed. A. Ubieto, Valencia 1966, 118. LUCAS DE TUY, Ibidem, p. 102. JIMÉNEZ DE RADA, Ibidem, VI, 32, ed. cit. p. 144. Primera Crónica General, II, p. 554 $n^{0} 884$.

${ }^{30}$ Los condes García Ordóñez y Martín Flaínez de León eran los principales apoyos de Alfonso VI en aquella época. El joven Gómez Martínez no era hijo de éste sino del ya difunto conde Martín Alfonso. En esto se equivoca B.F. REILLY (The Kingdom of León-Castilla under king Alfonso VI, Princeton, N.J. USA 1988, 354).

${ }^{31}$ JIMÉNEZ DE RADA, Ibidem, libro 7, cap. 15, p. 159. 
Por su parte los grandes genealogistas del siglo XVII nos brindan noticias disparatadas. Asentado que hubo dos condes con el nombre de García Ordóñez, el de Cabra y el de Nájera, al primero le asignan cinco hijos: García Ordóñez II, Diego Ordóñez, Fernando García, progenitor de los Zevallos, Gómez García y María Ordóñez. Y al segundo: García Garcés de Cabra y de Aza, y Nuño Garcés de Fuente Almejir ${ }^{32}$.

Vamos a exponer, con la ayuda de los diplomas y de una fuente musulmana, que hijos seguros del primer matrimonio fueron al menos tres: Fernando García, Mayor García y Elvira García. Se puede discutir si es hijo suyo Fortún Garcés Caixal, que fue teniente de Nájera.

\section{Fernando García de Hita}

Hace pocos años tratamos este misterioso personaje, al disertar sobre el fundador y primer maestre de la Orden de Santiago, Pedro Fernández, intentamos demostrar que su padre fue este Fernando García de Hita, e indicamos que una fuente musulmana hacía a éste "hijo del conde García" (Ordóñez): "No cabe duda, decíamos, que este Iben al-Zand Garsís, el hijo del conde García, es nuestro don Fernando García, que aparece como alcalde de las plazas de Medinaceli, Guadalajara e Hita" ${ }^{33}$.

Pero obsesionados con el prejuicio de que Fernando García tenía que ser hijo del rey García de Galicia, no prestamos fe a esta fuente árabe. Fue Jaime de Salazar Acha quien nos llamó la atención y puntualizó que la crónica árabe tenía razón: que el padre de Fernando García de Hita era el mismo conde García Ordóñez ${ }^{34}$.

$\mathrm{Y}$ en efecto, a pesar del silencio de la documentación y de las crónicas cristianas, creemos hoy razonable que el alcalde de Hita era hijo del conde García que murió en Uclés el año 1108. En el citado trabajo ya apuntamos los hijos de Fernando García. De su matrimonio con Tegridia (de la familia Ansúrez) le nacieron dos hijos ilustres: Gutierre Fernández de Castro, y Rodrigo Fernández de Castro. Es por tanto nuestro Fernando García cabeza de la Casa de Castro.

\footnotetext{
${ }^{32}$ SuÁREZ de Alarcón, Ibidem.

${ }^{33}$ IBN ABI-ZAR, Rawd al-Qirtás, ed. Huici Miranda, Valencia 1964.

${ }^{34}$ J.M. CANAL, Don Pedro Fernández, primer maestre de la Orden de Santiago, en “Anuario de est. Medievales”, 14 (1984), 33-71.
} 
Del segundo matrimonio con Estefanía Armengol, le nacieron cuatro hijos: Urraca Fernández, Martín Fernández, Sancha Fernández y Pedro Fernández, el fundador de la Orden de Santiago ${ }^{35}$.

En consecuencia, no hay motivo para identificar a este Fernando García con el conde Fernando, de quien habla la Historia Compostellana, a quien pide auxilio la misma reina Urraca Alfonso en la guerra del año 1110. Este conde pudo ser el conde Fernando Mitiz, tan silenciado por la documentación, pero que sabemos que existía el año 1080, año en que asistió al concilio de Burgos, con su hermano Nuño Mitiz, como ya hemos indicado ${ }^{36}$.

\section{Mayor García de Carrión}

Esta hija del conde García está bien documentada. Ella misma se nos presenta en un documento de Santa María la Real de Nájera, con fecha 1 de marzo de 1145: "Ego domna Maior, filia comitis Garsie et comitiisse domne Urrache, filiae regis Garsie, filii regis Sancii Maioris" ${ }^{37}$.

Aparece ella también en otra donación a San Juan de Burgos, con fecha 25 marzo 1140: "Ego domna Maior Garciez, filia comitis Garsie"38.

Estuvo casada con Gómez Martínez, hijo del conde Martín Alfonso, que murió en Uclés el año 1108, como ya indicado. La madre de este Gómez Martínez fue probablemente Elvira Gómez de Carrión, hija del conde Gómez Díaz de Carrión y Teresa Peláez de Asturias.

Este matrimonio de Mayor con Gómez Martínez se prueba con el documento de San Zoilo de Carrión, 18 mayo 1118, en que la misma doña Mayor, con sus hijos García Gómez, Pelayo Gómez y Diego Gómez, hace

\footnotetext{
${ }^{35}$ CANAL, Ibidem, 46-67.

${ }^{36}$ Identificamos a Fernando García, el hijo del conde García Ordóñez, con el conde Fernando, pariente de la reina Urraca, mencionado en la Historia Compostellana (libro I, cap. 63), pero esto fue un error evidente (ver mi art. cit. en nota 34 , p. 45).

Salazar Acha lo identifica con el dux Fernando Fernández que dona a Celanova, con su mujer Godo Pérez, el 22 noviembre 1108 (B.N. Mss. 834, fol. 411). Pero dudamos que este personaje fuese conde. Por lo cual habría que pensar más bien en el conde Fernando Mitid. De hecho, en el Cartulario de San Millán (ed. Serrano, p. 301) aparece un comes Ferrandus, en diploma con fecha 2 setiembre 1111 (ver también: SALAZAR ACHA, art. cit. supra en nota 12, p. 51$)$.

${ }^{37}$ Rodríguez de Lama, Colección de la Rioja, II, $2^{\text {a }}$ ed. p. 217, núm. 138.

${ }^{38}$ F.J. PEÑA PÉREZ, Documentación del monasterio de San Juan de Burgos, Burgos 1983, p. 23 , núm. 13.
} 
una donación por el alma de su marido "domni Gomiz (Martini) mariti mei" 39 .

Otra hija de Mayor y de Gómez fue Teresa Gómez, casada con García Fortunionis, que donan a Santa María de Nájera el año $1140^{40}$.

\section{Elvira García}

Que sea hija del conde García Ordóñez nos consta por un documento de la Orden de San Juan de Jerusalén, en el que el conde navarro Sancho Sánchez de Erro y de Tafalla menciona a su esposa: "ad meam mulierem, comitissa Alvira, filia de comite Garcea". Hija de estos fue María Sánchez, mujer de Diego López de Haro, señor de Vizcaya ${ }^{41}$.

\section{Fortún Garcés Kaixal (?)}

En abril de 1116 Alfonso I el Batallador hace una donación a su mayordomo, Fortún Garcés Kaixal, y luego, desde 1117 a 1133 le concede el gobierno y la tenencia de Nájera. ¿Cuál es la filiación de este misterioso personaje? Balparda, quizás por ese hecho de ocupar esa tenencia tanto tiempo, sospecha que sea hijo del conde García Ordóñez: "Sobre quién fuese, el dato de su parentesco con Sancho Ramírez de Aragón y sus hijos Pedro I y Alfonso I, el padre Moret (Annales II, 415) trae una donación suya a Cluny, del año 1135 por el alma de estos reyes, sus parientes, su mujer Toda y su hijo García, muerto en Mequinenza, reduce el número de familias en las que señalar su cuna. Pudiera ser hijo de García Ordóñez de Nájera y de la infanta su mujer"42.

\footnotetext{
${ }^{39}$ J.A. PÉREZ DE CELADA, Documentación del monasterio de san Zoilo de Carrión, Palencia, 986, I, p. 40, núm. 22. Notemos que entre los epitafios de Carrión, de ese monasterio propio de los Gómez de Carrión, está Gomez Martini, qui occisus est. Los cual indica que éste pertenecía a esta familia y esto podía ser sólo por parte de su madre. Notemos también que en ese pergamino del 18 de mayo de 1118 el apellido Martini está borrado, quizás por creer que era repetición de mariti mei. Y esto atendiendo que se cuenta aquí con abreviaciones. Datos sobre los Infantes de Carrión y sus epitafios en Sandoval (Historia de los Reyes).

${ }^{40}$ Rodríguez de lama, Colección de La Rioja, II, $2^{\text {a }}$ ed., p. 202, núm. 125.

${ }^{4 !}$ SalazAr ACHA, El Linaje de Castro, 54. Este conde Sancho de Erro hace una donación a la catedral de Pamplona, entre 1101 y 1104 , que edita J.M. Lacarra (Las Peregrinaciones a Santiago de Compostela, Pamplona 1992, III, p. 49).

${ }^{42} \mathrm{G}$. DE BALPARDA, Historia crítica de Vizcaya y de sus fueros, $2^{\mathrm{a}}$ ed., Bilbao 1974, II, 167 $\left(1^{\text {a }}\right.$ ed. I, 310).
} 
Contra esta conjetura está el hecho de que García Ordóñez estuvo siempre al servicio de los reyes de Castilla y de León, por lo que es muy difícil que un hijo suyo se afincase en un reino extraño. Es posible pero muy poco probable. Por otro lado sabemos que el Batallador repartía a dedo las mejores tenencias castellanas entre sus amigos y parientes.

\section{García Garcés de Aza}

Como hijos de su segundo matrimonio con la princesa Ava, podemos señalar solo a García Garcés, señor de Aza, de cuyo bautizo realizado el año 1106 en San Millán de la Cogolla ya hemos hablado.

El Toledano, al mencionar el parentesco de este personaje con los hijos del conde Pedro González de Lara, nos ofrece un dato interesante: "Hi tres (los condes Amalrico, Alvaro y Nuño Pérez de Lara) erant fratres, filii comitis Petri de Lara et Avae comitissae.Garsias Garsiae erat frater eorum ex matre, et filius comitis Garsiae, qui in bello Uclesii cum infante Sancio fuit occisus" ${ }^{43}$.

No vamos a insistir aquí sobre este personaje. Su historia aparece muy documentada, y ha sido ya descrita por autores como Julio González y Gonzalo Marínez Díez, a cuyos estudios remitimos ${ }^{44}$.

Este magnate es cabeza de la Casa de Aza y progenitor del glorioso patriarca santo Domingo de Guzmán, por lo cual ha sido muy mencionado por los biógrafos del santo castellano.

\section{CONCLUSIONES}

Como colofón de este artículo, será oportuno añadir algunas reflexiones sobre lo ya dicho.

\footnotetext{
${ }^{43}$ JIMÉNEZ DE RADA, Ibidem, libro 7, cap. 15, ed. Cabanes, p. 159.

${ }^{44}$ Julio GonZÁlez, El Reino de Castilla en la época de Alfonso VIII, Madrid 1960, I, 293. MARTínEZ DíEZ, art. cit. supra en nota 5, p. 186.
} 


\section{Valor de las fuentes}

Las fuentes para el conocimiento del conde García son, en parte, las mismas fuentes cidianas. Menéndez Pidal concedió a éstas mucho valor histórico, colocando incorrectamente la fecha de su composición. Sus conclusiones fueron estimadas como "definitivas" por el resto de los catedráticos españoles hasta hace muy poco. Modernos críticos como Colin Smith y B.F. Reilly se muestran intransigentes con él y corrigen muchas de esas conclusiones. Y no les falta razón.

Estos mismos autores han revalorizado la imagen del conde García hasta hace poco ensombrecida por la figura legendaria del $\mathrm{Cid}^{45}$.

\section{Ascendencia del conde}

Los marcos del presente estudio no nos han permitido explicar a fondo este problema. Añadamos que la tesis de que Ordoño Ordóñez, el padre del conde, procede de la Casa Real leonesa, o mejor, astur leonesa, lleva las de ganar sobre la tesis del origen castellano, asentada por Menéndez Pidal. Las razones expuestas por Sánchez Candeira tienen su peso. Y a ellas remitimos.

\section{Sobre la descendencia}

El silencio incomprensible de los autores cristianos sobre este punto ha sido reparado por las fuentes musulmanas. Fernando García, el hombre de la frontera, que sucedió en esta difícil tarea al valeroso caudillo Alvaro Fáñez, el valioso colobarador de la reina Urraca, como atestigua el Anónimo I de Sahagún, no será hijo del rey García de Galicia, como se ha creído

\footnotetext{
${ }^{45}$ Como ejemplo de esa aceptación general de las tesis pidalianas, pueden verse varios artículos en Historia general de las literaturas hispánicas, Barcelona 1949, I, publicadas bajo la dirección de G. Díaz Plaja, con la introducción de R. MENÉNDEZ PIDAL. Claro que hubo varias excepciones, como la del profesor aragonés A. Ubieto, ya citado supra en nota 1. Tampoco queremos decir que estemos de acuerdo con todas las afirmaciones de SMITH y de REILLY, cuyas obras hemos citado en notas 4 y 30 . Ya hemos dicho que REILLY se equivoca al decir que el Gómez Martínez que murió en Uclés, 1108, era hijo del conde Martín Flaínez. También se equivoca, pensamos, al asertar que en los diplomas auténticos están presentes en la Cancillería o en la Curia todos los confirmantes y testigos. Aquí le contradecimos, y damos la razón al padre Avelino de Jesús da Acosta (Dedicaçao da Sé de Braga, 28 agosto 1089. Risposta a B. Reilly, Braga 1991, 36 pp.). Varios documentos de Alfonso VI son postdatarios y contienen por ende errores sobre personas y sobre las fechas, aún siendo auténticos.
} 
tradicionalmente, sino del conde García. El es "el hijo del conde García", afirma la crónica árabe, sin titubear.

Y por tanto, al conde García cabe la gloria de ser cabeza de la ilustre Casa de Castro, y al mismo tiempo la cabeza de la Casa de Aza, progenitora del glorioso Santo Domingo de Guzmán.

\section{El apéndice documental}

Ponemos en primer lugar una donación del conde García, cuya transcripción nos ha proporcionado gentilmente el canónigo de Pamplona don José Goñi. Son muchos los documentos que mencionan al conde, pero muy pocos los otorgados por él mismo. Por eso nos complacemos en presentar aquí este texto de un archivo navarro.

El segundo documento es muy interesante. No sólo porque ilustra la figura del conde Fernando Mitiz, que en otro anterior estudio confundimos con el hijo del conde, Fernando García, sino porque es desconocido en España y nos da un dato curioso sobre la obsidio, el cerco musulmán o almorávide, que la ciudad de Toledo sufrió en el año 1118, al que concurrieron tropas gallegas ${ }^{46}$.

El tercero puede ilustrar la familia de ese magnate al servicio de Alfonso I el Batallador, que fue Fortún Garcés, a quien algunos quieren hacer hijo del conde García. El erudito lector puede examinarlo de cerca y juzgar por sí mismo. Nos inclinamos a pensar que no es hijo de nuestro conde.

${ }^{46}$ Advierto que este documento, ya citado por L. SERRANO, no lo hemos encontrado ni en la Biblioteca Nacional, ni en el Centro de Estudios Históricos, de Madrid. Mi sincero agradecimiento al citado padre Avelino y a la señora profesora de la Universidad de Coimbra que muy amablemente me lo proporcionaron. 


\section{APÉNDICE DOCUMENTAL}

1084, agosto.

El conde García (Ordónez) y su mujer la condesa Urraca donan al monasterio de San Adrián (Navarra) varias tierras y viñas en Azagra, Sotolga, Almonasterio, Valdillo de Rey, Pesencanos, Campo de Benesat, Soto de Olivo, Pinilla, Calahorra, Arresa, Almonacer y Oyón, y encomiendan la regencia de la iglesia a don García, clérigo de Almonasterio.

Pamplona, A. Catedral, Libro redondo, f. 152

(copia siglo XIII, falta un trozo).

Publ.: J. GoÑI GAZTAMBIDE, Los obispos de Pamplona del siglo XII, "Anthologica

Annua", 13 (1965), 325.

(CHristus). Sub Christi nomine. Ego Gassea, gratia Dei comes, simul cum dilecta coniuge Urraca comitissa, nullius cogentis imperio nec suadentis ingenio, sed pro amore Domini nostri Ihesu, tota devotione mentis et corporis facimus hoc testamentum, legali auctoritate et testimonio fidelissimorum hominum roboratum inconcusso robore et tenore.

Denique cum multis facinoribus nos agnoscimus esse prepeditos et mundialibus curis deditos, nullo nostro merito confidimus posse salvari, nisi sanctorum patrocinio et continua eorum intercessione. Ideoque plerumque relatione, ut agnovimus, qualiter sine reverentia erat dedita ecclesia Sancti Adriani martiris, que est sita in ripa qui dicitur Palma, iuxta Ibero flumine, cui iungitur ex adverso Ega flumen, ubi multa miraculorum signa, multeque concurrentium curationes eodem prefato martire interpellante declarantur, indignum duce (?) procuravimus talem locum absque clericorum frequentatione manere... gubernatorem dompnum Garseanum de Almonasterio... consistat... datur vel fuerit sive totum quod a ceteris fidelibus... et luminariis frequentetur, prout potuerit... eam dimittere, dimittat suo nepoti vel aliorum clericorum qui dignum... rege sicut et ipse. Donamus namque et in Azahra subtus villam et sub... limite de Fortunio Santii, et inter limites de vicinos unam vineam... in Sotolga, iuxta rivo, quod aperuerunt vicinos. In Almonasterio illam... Vadiello de Rege, et una terra ante Sancti Adriani. In Pesencano... 
Dominico Santio, et aliam in Campo de Benesat. In Soto de Olivo unam terram vineam, unam vineam in Penella, et illas de Calagorra et Arresa et Alomonazer et Açahra et Ohion totas integras.

$\mathrm{Si}$ quis hoc nostrum testamentum in aliquo perturbaverit, maledictio Dei et omnium Sanctorum eius veniat super eum hic et in futuro, et exsolvat regi X libras auri, et quod compulsavit componat iam dicte ecclesie meliori duplo.

Santius espiscopus confirmat. Senior Eneco Acenariz testes. Domino Apparitio testes. Dominico Santii testes. Garsia Santii testes. Fortunio Ioannis testes. Garcia Orioli testes. Concilio de Calagorra testes. Azahra et Almonasterio et Arresa testes.

$(+)$.

Ego Adefonsus, Dei gratia rex, hanc cartam laudo et manu mea confirmo

Era $\mathrm{M}^{\mathrm{a}} \mathrm{C}^{\mathrm{a}} \mathrm{XXII}{ }^{\mathrm{a}}$, die $\mathrm{II}^{\mathrm{a}}$ Feria mediante augusto. Eneco notavit et signavit.

1118, septiembre, 20.

Alfonso Vll, con el consentimiento de su madre la reina Urraca Alfonso, dona a Gómez Núñez todos los bienes que su abuela (de éste) la condesa Goncina, y su tío el conde Fernando Midiz habían poseído en la tierra de Toroño.

Biblioteca Gen. de la Univ. de Coimbra (Portugal), Mss. 731, nº 148

(Documentos para a Historia Portuguesa), p. 122.

Nota: Es copia de un documento del Cartulario del monasterio de Pombeiro (Lugo).

CHRISTUS. Ego Adefonsus, gratia Dei rex Hispaniae, comitis Raimundi filius, consentiente matre mea, bonae memoriae regis Adefonsi filia, regina videlicet domna Urraca, facio vobis Gumez Nuniz kartam donationis de omnibus illis haereditatibus, quae fuerunt de avia tua comitissa domna Gumcina et de tio tuo comite domno Fernando Midiz, in tota illa terra, quae vos tenetis de me in Toronio, videlicet cum omni criatione, scilicet servos et ancillas et iuniores et benefacturia. Et do vobis villas tam laicales quam monesteriales, heremas et pobladas, parochias et heremitas, sicuti eas melius unquam habuit aliquis homo de gente tua, vel de extranea. Et facio vobis talem fiduciam, quod si Deus michi illam terram in quacumque haereditates fuerint de tuis parentibus, dederit, et totas illas vobis promitto pro bono servitio et grata fidelitate, quam nobis tenuisti, ad faciendum de ea quaecumque vestra et omni progeniei vestrae, vel cui illas dare ver vendere volueris, aevo perenni. 
Si quis vero de nostra gente vel de extranea hoc factum nostrum violare, vel aliquo modo infringere voluerit, quisquis ille fuerit, imprimis sit excommunicatus, et cum Iuda, Domini proditore, in inferno cruciatus.

Facta haec karta in Socobia, redeunte regina domna Urraca cum filio suo rege domno Adefonso a Toletana obsidione, cum galliciano exercitu, sub Era M.C. LVI, et quotum XII Kalendarum Octobrium.

Regnante regina domna Urraca cum filio suo rege Adefonso in civitate Legionensi.

Et si quis ausus fueret infringere, pariat vobis hanc vecem intendenti C. libras purissimi auri.

(Col. $1^{\text {a }}$ ) Ego rex Adefonsus, quod fieri iussi confirmo (signo, y dentro del mismo: ADEFONSUS).

Ego comes P. Froilaci cf.; Ego comes G. Vermudiz cf.; Ego A. comes $\left(\right.$ Col 2 ${ }^{\text {a }}$ ) Ego Rudericus Petrici, comitis Petri filius cf.; Ego Ansur, comitis Sancii filius cf., Ego Pelagius Sudarici cf.; Ego Arias Petrici cf. (Col. $3^{\text {a }}$ ) Ego B. Toletanae Sedis Archiepiscopus cf.; Ego G. Salmantinae Sedis eps. cf.; Ego Daniel, regis capellanus cf.; Ego P., regis notarius, quod scripsi robore (+).

1145

Fortún Garcés Caixal dona a Cluny la iglesia de San Adrian de Vadoluengo.

París, B.N., or. 238 y 239; Coll. Bourgogne 76-85.

Publ.: A. BRuEl, Recueil des chartes de l'abbaye de Cluny, V, p. 453, nº 4104.

In Christi nomine et eius divina clementia. Ego Fortunio Garceiz Kaixal facio hanc cartam donationis. Dono et concedo Deo et sancto Petro Cluniacensi ecclesiam beati Adriani de Vado Longo, cum illa hereditate quam desponsavimus ego et uxor mea Tota die consecrationis eius (ecclesiae). Dono insuper molendinum et vineam que est circa palatium et agrum qui dicitur Peculiare, qui est supra pontem in Urzella, et hortum ubi puteus est, ad caput ecclesie, et in burgo novo palatium meum, et totam ipsam hereditatem, quam Cluniacenses monachi iam tenebant et possidebant, ex die consecrationis prenominate ecclesie, quando ego et predicta uxor mea Tota obtulimus illam prefato Sancto Petro, in manu et in presentia Sancii, Pampilonensis episcopi.

Hoc autem donativum facio propter salutem et remedium anime mee vel parentum meorum, regis Sancii et filiorum eius, regis Petri et Aldefonsi, et uxoris 
mee Tote, atque filii mei Garcie, ut sit in salutem et remedium animarum nostrarum, per secula cuncta. Amen.

Dono autem sic ut habeant et possideant predicti monachi ecclesiam beati Sanctii Adriani, cum omnibus prefatis hereditatibus ingenuam et liberam et quietam et sine ulla mala voce, per secula cuncta. Amen.

Quicumque vero ex nepotibus vel ex generatione nostra, hoc donativum violare aut frangere voluerit, sit anathema et excomunicatus, et cum Datam et Abiron et cum Iuda traditore sepeliatur in inferno.

De hoc sunt testes: Ioannes abbas de Sancti Ioannis de Pinna, Petrus abbas sancti Salvatoris Leirensis cenobii, Petrus abbas Sancte Marie de Irax. Et in Burgo Novo de Sangossa, ubi palatium est cum sua exita directa usque ad terminum coram vicinis statutum. Sunt testes: Gilermus Cegorra, Gilermus Ugo, Sancius Picator, Miletus Bivianus, frater eius. Arnaldus vel Nicholaus faber, Ioannes Motila, Petrus de Lafita, Gilermus Ross de Uli, García Semenonis, Ferrann Galinz, Lope Arceiz de Sancto Adriano, Eneco Eneconis, Petrus Castellanus, Petrus Arnaldi, Blasco Sancte Eulalie.

SIGNUM + CAIXAL

Facta carta Era $\mathrm{M}^{\mathrm{a}} \mathrm{C}^{\mathrm{a}} \mathrm{LXXX}^{\mathrm{a}} \mathrm{III}^{\mathrm{a}}$, regnante domino nostro Iesu Christo, et sub eius imperio dominante comite Barchinonensi ac principante in Aragone et in Sobarbe et in Ripacurtia, in Cesaraugusta et in Calatavibus. Regnante rege Garcia in Pampilone et Alava et Biscaia et in Tutela. Episcopo Lope, cuius interventu et auctoritate hanc cartam facio, existente animarum procuratore in Pampilona. Episcopo Dodone in Osca, episcopo Michaele in Tarassona, episcopo Sancio in Calahorra et in Nagera. Fiat, fiat.

Ego Petrus, prefati Kaixal capellanus, licet indignus, hanc cartam scripsi et hoc signum + posui.

\section{RÉSUMÉ}

Le comte García Ordoñez apparais comme le rival du Cid Campeador dans les ouvrages litteraires que louent ce dernier. Ces ouvrages sont: Historia Ruderici Campidocti, Carmen Campidoctoris et le Cantar de Mio Cid. Au même time, logiquement, tentent de dénigrer le comte García mélangeant éléments historiques avec élements fabuleux. Les auteurs des Cronicas Generales tombèrent dans le piège et présentaient comme histoire ce que était plus qu'une fiction. Cet article tente de démentir la légende noire et présenter la personne historique du comte, utilisant documents dignes de foi: on parle de ses ancêtres, son épouse, ses fils, sa loyauté au roi Alfonse VI, sa participation à la Reconquête, son pas par trahison aux Maures, sa chûte en disgrâce du roi, sa inaptitude comme stratège, etc. 


\section{SUMMARY}

Count García Ordóñez appears in literary works exalting the Cid Campeador as his rival. The works concerned are Historia Ruderici Campidocti, Carmen Campidoctoris and the Cantar de Mio Cid. Logically, they also attempt to undermine the figure of Count Garcia by mixing historical and fictitious elements. The authors of the Crónicas Generales fell into the trap and presented these elements as historical fact. This brief article tries to show the historical figure of the Count: his ancestors, wives, children, loyalty to Alfonso VI, his courageous participation in the Reconquest, at the same time demolishing the black legend: his support of the Moors, the king's anger towards him, his ineptitude as a strategist, etc. 